\title{
Value of Neutrophil to Lymphocyte Ratio in Early Prediction of Meconium Aspiration Syndrome
}

\author{
Suzan Gad ${ }^{1}$ Abeer Alkhalafawi ${ }^{2}$ Syed Raza ${ }^{3}$ Mervat Hesham ${ }^{4}$ Mohamed Sheta ${ }^{5}$
}

${ }^{1}$ Department of Pediatrics, Faculty of Medicine, Suez Canal
University, Ismailia, Egypt
2 Department of Pediatrics, Ras Elkheima Hospital, Ras Elkheima, UAE
${ }^{3}$ Department of Pediatrics, Sheikh Khalifa Medical City, Ajman, UAE
${ }^{4}$ Department of Pediatrics, Faculty of Medicine, Zagazig University,
Zagazig, Egypt
${ }^{5}$ Department of Pediatrics, Mansoura General Hospital, Mansoura, Egypt

J Child Sci 2020;10:e207-e211.

\author{
Address for correspondence Mohamed Sheta, MRCPCH (UK), MSc \\ (Pediatrics), DCH, Department of Pediatrics, Mansoura General \\ Hospital, Mansoura, Egypt (e-mail: sheta99999@yahoo.com).
}

\begin{abstract}
Keywords

- meconium

- meconium aspiration

- meconium aspiration syndrome

- neutrophil to lymphocyte ratio

Meconium aspiration syndrome (MAS) is a serious neonatal condition. Prediction of MAS is challenging particularly in low-resource setting. Neutrophil to lymphocyte ratio (NLR) is a new simple index used for diagnosis of many inflammatory conditions. The present study was an attempt to determine whether NLR can predict the occurrence of MAS. The present study included 101 children with meconium-stained amniotic fluid. They comprised 22 patients who developed MAS and 79 neonates who didn't have the condition. All neonates were subjected to careful analysis of maternal and perinatal history in addition to thorough clinical assessment and radiological and laboratory evaluation. Blood samples from the umbilical cord were collected at birth and were used to obtain complete blood counts and C-reactive protein (CRP) assay. Univariate analysis revealed that elevated CRP levels, increased NLR, and 5-minute Apgar score of $<7$ were significant risk factors for the occurrence of MAS. Multivariate analysis revealed that low 5-minute Apgar score and increased NLR remained as significant risk factors of MAS. Receiver operating characteristic curve analysis showed good performance of NLR in prediction of MAS. NLR is useful in prediction of MAS in term neonates with meconium-stained amniotic fluid.
\end{abstract}

\section{Introduction}

Meconium aspiration syndrome (MAS) is a neonatal lung injury induced by meconium aspirated into the lung and airways. $^{1}$ It is associated with significant mortality ${ }^{2}$ and morbidities including persistent pulmonary hypertension and pneumonitis ${ }^{3,4}$ particularly in developing countries. ${ }^{5-7}$ It is crucial to identify infants at high risk of acquiring MAS and to distinguish cases with severe disease. This constitutes the cornerstone of effective prevention and treatment strategy in low-resource setting. ${ }^{8}$

It is thought that fetal systemic inflammation is an important pathological mechanism in the development of MAS. ${ }^{9}$ Labora-

received

July 13,2020

accepted after revision

September 28, 2020
DOI https://doi.org/

10.1055/s-0040-1720958. ISSN 2474-5871. tory markers investigated to assess the role of inflammation in MAS included C-reactive protein (CRP) levels, immature-tototal neutrophil (IT) ratio, ${ }^{10}$ and toll-like receptors levels. ${ }^{11}$

Neutrophil to lymphocyte ratio (NLR) is a novel marker of inflammation that was extensively investigated in a wide range of medical conditions affecting adults ${ }^{12-15}$ and children. ${ }^{16-19}$ In neonates, increased level of NLR was related to patency of patent ductus arteriosus in preterm infants, ${ }^{20}$ low survival in patients with solid tumors, ${ }^{21}$ neonatal sepsis, ${ }^{22}$ and neonatal necrotizing colitis. ${ }^{23}$ The present study aims to assess the value of NLR in the prediction of MAS and its relation to disease severity.
Copyright @ 2020 Georg Thieme Verlag License terms
KG Stuttgart · New York 


\section{Materials and Methods}

The present study was a prospective case-control study. It was conducted at Pediatric Department, Mansoura General Hospital, Mansoura, Egypt. All singleton term neonates with meconium-stained amniotic fluid (MSAF) at delivery in the period from January, 2013 through December, 2017 were included in the study. Among them, neonates with MAS were diagnosed on the basis of clinically manifest respiratory distress within 24 hours after birth and radiologically evident pneumonitis or air-trapping. Neonates with congenital anomalies and those with risk factors or evidence of neonatal sepsis were excluded from the study.

All neonates were subjected to careful analysis of maternal and perinatal history in addition to sophisticated clinical assessment and radiological and laboratory evaluation. Blood samples from the umbilical cord during the 1st hour after delivery were used to obtain complete blood counts and CRP assay.

All the 117 neonates included in the study had MSAF at recruitment. However, 16 of those neonates were excluded from the study because they had one or more of the exclusion criteria. Among the remaining 101 neonates, those who later developed MAS $(n=22)$ were considered as the study patients, while the 79 neonates who didn't have the condition served as controls. Patients with MAS were classified as having severe disease if they needed assisted ventilation for more than 48 hours or nonsevere disease if they required such intervention for less than 48 hours. $^{24}$

Informed consent was obtained from the legal guardians of all participants and the study was conducted according to the guidelines of the Declaration of Helsinki and was approved by the local ethical committee.

Statistical analysis was achieved using SPSS, version 22 (IBM, Armonk, New York, United States). Categorical variables were expressed as number and percent, while continuous data were represented as mean and standard deviation. Student's $t$-test was used for continuous data comparisons, while Fisher's exact test was used to compare categorical data. Logistic regression analysis was performed to reveal predictors of MAS. Only significant factors in univariate analysis were advanced into multivariate analysis. Receiver operating characteristic (ROC) curve analysis was utilized to assess the diagnostic value of NLR. A p-value less than 0.05 was considered statistically significant.

\section{Results}

The present study included 22 neonates with MAS and 79 neonates with MSAF. The latter group served as control group. Comparison between both groups regarding the basic laboratory data is illustrated in - Table 1. Patients with MAS had significantly higher CRP levels ( $14.7 \pm 7.5$ vs. $2.3 \pm 1.9 \mathrm{mg} / \mathrm{dL}$, $p<0.001)$ and NLR $(2.03 \pm 0.72$ vs. $1.33 \pm 0.35, p<0.001)$

Table 1 Comparison between neonates with MAS and without regarding the basic data

\begin{tabular}{|c|c|c|c|}
\hline & $\begin{array}{l}\text { MAS } \\
n=22\end{array}$ & $\begin{array}{l}\text { MSAF without MAS } \\
n=79\end{array}$ & $p$-Value \\
\hline \multicolumn{4}{|l|}{ Sex, $n(\%)$} \\
\hline Male & $12(54.5)$ & $39(49.4)$ & 0.677 \\
\hline Female & $10(45.5)$ & $40(51.6)$ & \\
\hline Gestational age (wk) mean \pm SD & $37.9 \pm 2.1$ & $38.1 \pm 1.7$ & 0.658 \\
\hline Birth weight $(\mathrm{kg})$ mean $\pm S D$ & $3.3 \pm 0.4$ & $3.5 \pm 0.4$ & 0.064 \\
\hline \multicolumn{4}{|l|}{ Mode of delivery, $n(\%)$} \\
\hline Vaginal & $13(59.1)$ & $41(51.9)$ & 0.550 \\
\hline CS & $9(40.9)$ & $38(48.1)$ & \\
\hline Fetal distress, $n(\%)$ & $7(31.8)$ & $16(20.3)$ & 0.25 \\
\hline Pulmonary hypertension, $n(\%)$ & $15(68.2)$ & - & $<0.001$ \\
\hline $\mathrm{Hb}(\mathrm{gm} / \mathrm{dL})$ & $17.7 \pm 2.5$ & $18.4 \pm 1.8$ & 0.108 \\
\hline Platelets $\left(\times 10^{\wedge} 3 / \mathrm{mL}\right)$ mean $\pm \mathrm{SD}$ & $260.0 \pm 76.7$ & $291.1 \pm 82.8$ & 0.117 \\
\hline WBCs $\left(\times 10^{\wedge} 3 / \mathrm{mL}\right)$ mean $\pm S D$ & $14.6 \pm 3.7$ & $16.0 \pm 3.8$ & 0.107 \\
\hline $\mathrm{CRP}(\mathrm{mg} / \mathrm{dL})$ mean $\pm \mathrm{SD}$ & $14.7 \pm 7.5$ & $2.3 \pm 1.9$ & $<0.001$ \\
\hline NLR mean $\pm S D$ & $2.03 \pm 0.72$ & $1.33 \pm 0.35$ & $<0.001$ \\
\hline Oxygen therapy, $n(\%)$ & $22(100.0)$ & $6(7.6)$ & $<0.001$ \\
\hline Mechanical ventilation, $n(\%)$ & $9(40.9)$ & 7 (8.9) & $<0.001$ \\
\hline Surfactant therapy, $n$ (\%) & $6(27.3)$ & - & $<0.001$ \\
\hline 5 minutes Apgar score $<7, n(\%)$ & $13(59.1)$ & $11(13.9)$ & $<0.001$ \\
\hline
\end{tabular}

Abbreviations: CRP, C-reactive protein; CS, cesarean section; Hb, hemoglobin; MAS, meconium aspiration syndrome; MSAF, meconium-stained amniotic fluid; NLR, neutrophil lymphocyte ratio; SD, standard deviation; WBCs, white blood cells.

Data presented as mean \pm SD or number and percent. 
when compared with controls. Also, patients had significantly higher rates of low Apgar score (59.1 vs. 13.9, $p<0.001$ ), oxygen therapy ( 100.0 vs. $7.6 \%, p<0.001$ ), and mechanical ventilation ( 40.9 vs. $8.9 \%$ ). MAS patients included 8 patients (36.4\%) with severe MAS and the remainder 14 patients (63.6\%) presented with nonsevere MAS.

Logistic regression analysis for predictors of MAS in the present study revealed that on univariate analysis, elevated CRP levels, increased NL ratio, and 5 minutes Apgar score $<7$ were significant predictors of MAS. However, only low Apgar score (odds ratio [OR] [95\% confidence interval (CI)]: 0.1 [0.01-0.94]) and NLR (OR [95\% CI]: 0.61 [0.49-0.78]) remained significant using multivariate analysis (-Table 2). ROC analysis shows a good value of NLR in the diagnosis of MAS (area under the curve: 0.86 , sensitivity: $81.8 \%$, specificity: $79.5 \%)$. However, NLR failed to predict severity of MAS (-Table 3, - Fig. 1).

\section{Discussion}

The present study provides the first report of using the simple NLR in the prediction of MAS in absence of concomitant septicemia. In our study, NLR showed significant value in prediction of MAS both in univariate and multivariate analysis with a good sensitivity and specificity. In comparison, the work of Hofer et $\mathrm{al}^{10}$ assessed the relationship between absolute neutrophil count and IT ratio in addition to CRP and MAS. They found significant association between elevated IT ratio and CRP levels and MAS severity. However, their study

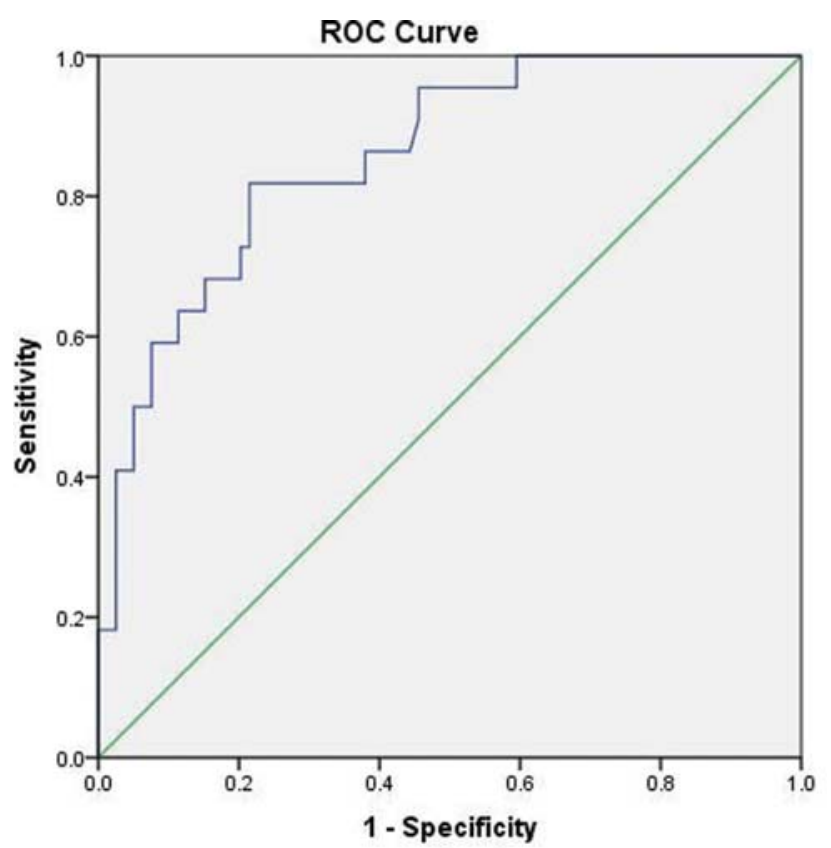

Diagonal segments are produced by ties.

Fig. 1 Receiver operating curve (ROC) showing value of neutrophil to lymphocyte ratio in the diagnosis of meconium aspiration syndrome (area under the curve: 0.86 , sensitivity: $81.8 \%$, specificity: $79.5 \%$ ).

Table 2 Univariate and multivariate regression analysis for predictors of MAS in the studied patients

\begin{tabular}{|l|l|l|l|l|l|l|}
\hline & \multicolumn{4}{|l|}{ Univariate analysis } & \multicolumn{4}{l|}{ Multivariate analysis } \\
\cline { 2 - 7 } & OR & Cl & $p$-Value & OR & CI & $p$-Value \\
\hline Sex & 0.81 & $0.32-2.1$ & 0.668 & - & - & - \\
\hline Gestational age & 1.01 & $0.82-1.4$ & 0.655 & - & - & - \\
\hline Birth weight & 2.8 & $0.92-8.8$ & 0.071 & - & - & - \\
\hline Mode of delivery & 0.75 & $0.29-1.9$ & 0.550 & - & - & - \\
\hline Hb & 1.2 & $0.95-1.6$ & 0.111 & - & - & - \\
\hline Platelets & 1.0 & $0.99-1.0$ & 0.119 & - & - & - \\
\hline WBCs & 1.1 & $0.97-1.3$ & 0.110 & - & - & - \\
\hline CRP & 0.6 & $0.49-0.73$ & $<0.001$ & 0.83 & $0.08-8.2$ & 0.87 \\
\hline NLR & 0.17 & $0.06-0.47$ & $<0.001$ & 0.61 & $0.49-0.78$ & $<0.001$ \\
\hline 5 minutes Apgar score $<7$ & 0.11 & $0.04-0.32$ & $<0.001$ & 0.1 & $0.01-0.94$ & 0.044 \\
\hline
\end{tabular}

Abbreviations: $\mathrm{Cl}$, confidence interval; CRP, C-reactive protein; MAS, meconium aspiration syndrome; NLR, neutrophil to lymphocyte ratio; OR, odds ratio; WBCs, white blood cells.

Table 3 Value of NLR in diagnosis and grading of MAS

\begin{tabular}{|l|l|l|l|l|l|}
\hline & Cutoff & AUC & $p$-Value & Sensitivity & Specificity \\
\hline Diagnosis (MAS vs. MSAF) & 1.52 & 0.86 & $<0.001$ & $81.8 \%$ & $79.5 \%$ \\
\hline Grading (severe vs. nonsevere) & 2.01 & 0.74 & 0.065 & $62.5 \%$ & $85.7 \%$ \\
\hline
\end{tabular}

Abbreviations: AUC, area under the curve; MAS, meconium aspiration syndrome; MSAF, meconium-stained amniotic fluid; NLR, neutrophil to lymphocyte ratio. 
didn't include control group and so, the diagnostic value of these markers wasn't assessed.

Use of NLR in the diagnosis of inflammatory neonatal complications in the absence of microbial infection was previously reported. In the study of Kurtul et al, ${ }^{25}$ neonates with retinopathy of prematurity showed significantly higher NLR when compared with controls.

The lower lymphocyte counts in MAS patients in our study may be explained by the exaggerated response to the steroid surge of labor ${ }^{12,26}$ in which the role of lymphocyte corticosteroid receptors was implicated. ${ }^{27-29}$ The diminished lymphocyte population could result in enhanced inflammatory process by reducing expression of intereukin-10; the main anti-inflammatory cytokine detected in patients with MAS and rise of the proinflammatory cytokines including intereukin-6, tumor necrosis factor $\alpha$, and interferon gamma. ${ }^{30}$ However, these mechanisms remain to be elucidated.

The present study has the strength of its prospective casecontrol design and long study period. However, our conclusions are limited by the relatively small sample size. From the practical point of view, performing differential leucocytic count is a widely available investigation even in low-resource settings and its use to distinguish neonates with MAS in the critical window of the first postnatal hour would provide a paramount help to the decision making in these situations. Findings of the present study are limited by the lack of cord blood gases analysis.

\section{Conclusion}

NLR is a useful tool for early prediction of MAS in term neonates with MSAF in the absence of sepsis/infection.

\section{Funding}

None.

\section{Conflict of Interest}

None declared.

\section{References}

1 Piastra M, Yousef N, Brat R, Manzoni P, Mokhtari M, De Luca D. Lung ultrasound findings in meconium aspiration syndrome. Early Hum Dev 2014;90(Suppl 2):S41-S43

2 Louis D, Sundaram V, Mukhopadhyay K, Dutta S, Kumar P. Predictors of mortality in neonates with meconium aspiration syndrome. Indian Pediatr 2014;51(08):637-640

3 Choudhary M, Meena MK, Chhangani N, Sharma D, Choudhary JS, Choudhary SK. To study prevalence of persistent pulmonary hypertension in newborn with meconium aspiration syndrome in western Rajasthan, India: a prospective observational study. J Matern Fetal Neonatal Med 2016;29(02):324-327

4 Shaikh M, Irfan Waheed KA, Javaid S, Gul R, Hashmi MA, Fatima ST. Detrimental complications of meconium aspiration syndrome and their impact on outcome. J Ayub Med Coll Abbottabad 2016;28(03):506-509

5 Chettri S, Bhat BV, Adhisivam B. Current concepts in the management of meconium aspiration syndrome. Indian J Pediatr 2016;83 (10):1125-1130

6 Panton L, Trotman H. Outcome of neonates with meconium aspiration syndrome at the university hospital of the West Indies,
Jamaica: a resource-limited setting. Am J Perinatol 2017;34(12): $1250-1254$

7 Lama S, Mahato SK, Chaudhary N, et al. Clinico-radiological observations in meconium aspiration syndrome. JNMA J Nepal Med Assoc 2018;56(209):510-515

8 Karabayir N, Demirel A, Bayramoglu E. Blood lactate level and meconium aspiration syndrome. Arch Gynecol Obstet 2015;291 (04):849-853

9 Lee J, Romero R, Lee KA, et al. Meconium aspiration syndrome: a role for fetal systemic inflammation. Am J Obstet Gynecol 2016; 214(03):366.e1-366.e9

10 Hofer N, Jank K, Strenger V, Pansy J, Resch B. Inflammatory indices in meconium aspiration syndrome. Pediatr Pulmonol 2016;51 (06):601-606

11 Anand V, Basu S, Yadav SS, Narayan G, Bhatia BD, Kumar A. Activation of toll-like receptors in meconium aspiration syndrome. J Perinatol 2018;38(02):137-141

12 Balta S, Celik T, Mikhailidis DP, et al. The relation between atherosclerosis and the neutrophil-lymphocyte ratio. Clin Appl Thromb Hemost 2016;22(05):405-411

13 DiGangi C. Neutrophil-lymphocyte ratio: predicting cardiovascular and renal complications in patients with diabetes. J Am Assoc Nurse Pract 2016;28(08):410-414

14 Mazza MG, Lucchi S, Tringali AGM, Rossetti A, Botti ER, Clerici M. Neutrophil/lymphocyte ratio and platelet/lymphocyte ratio in mood disorders: a meta-analysis. Prog Neuropsychopharmacol Biol Psychiatry 2018;84(Pt A):229-236

15 Wang Y, Peng C, Cheng Z, et al. The prognostic significance of preoperative neutrophil-lymphocyte ratio in patients with hepatocellular carcinoma receiving hepatectomy: a systematic review and meta-analysis. Int J Surg 2018;55:73-80

16 Dogru M, Evcimik MF, Cirik AA. Is neutrophil-lymphocyte ratio associated with the severity of allergic rhinitis in children? Eur Arch Otorhinolaryngol 2016;273(10):3175-3178

17 Yigit Y, Yilmaz S, Akdogan A, Halhalli HC, Ozbek AE, Gencer EG. The role of neutrophil-lymphocyte ratio and red blood cell distribution width in the classification of febrile seizures. Eur Rev Med Pharmacol Sci 2017;21(03):554-559

18 Dogru M, Citli R. The neutrophil-lymphocyte ratio in children with atopic dermatitis: a case-control study. Clin Ter 2017;168 (04):e262-e265

19 Hüner EA, Dai AI, Demiryürek AT. Association of neutrophil/ lymphocyte ratio with intravenous immunoglobulin treatment in children with Guillain-Barré syndrome. J Child Neurol 2018;33 (02):164-167

20 Temel MT, Coskun ME, Akbayram S, Demiryürek AT. Association between neutrophil/lymphocyte ratio with ductus arteriosus patency in preterm newborns. Bratisl Lek Listy 2017;118(08): 491-494

21 Nayak A, McDowell DT, Kellie SJ, Karpelowsky J. Elevated preoperative neutrophil-lymphocyte ratio is predictive of a poorer prognosis for pediatric patients with solid tumors. Ann Surg Oncol 2017;24(11):3456-3462

22 Omran A, Maaroof A, Saleh MH, Abdelwahab A. Salivary C-reactive protein, mean platelet volume and neutrophil lymphocyte ratio as diagnostic markers for neonatal sepsis. J Pediatr (Rio J) 2018;94 (01):82-87

23 Yang Y, Cao ZL, Zhou XY, et al. Does neutrophil/lymphocyte ratio have good diagnostic value in neonatal necrotizing colitis? J Matern Fetal Neonatal Med 2018:1-8. Doi: 10.1080/14767 058.2018.1455182

24 Cleary GM, Wiswell TE. Meconium-stained amniotic fluid and the meconium aspiration syndrome. An update. Pediatr Clin North Am 1998;45(03):511-529

25 Kurtul BE, Kabatas EU, Zenciroglu A, et al. Serum neutrophil-tolymphocyte ratio in retinopathy of prematurity. J AAPOS 2015;19 (04):327-331 
26 Stjernholm YV, Nyberg A, Cardell M, Höybye C. Circulating maternal cortisol levels during vaginal delivery and elective cesarean section. Arch Gynecol Obstet 2016;294(02):267-271

27 Krüger K, Agnischock S, Lechtermann A, et al. Intensive resistance exercise induces lymphocyte apoptosis via cortisol and glucocorticoid receptor-dependent pathways. J Appl Physiol (1985) 2011; 110(05):1226-1232

28 Vianna P, Bauer ME, Dornfeld D, Chies JA. Distress conditions during pregnancy may lead to pre-eclampsia by increasing corti- sol levels and altering lymphocyte sensitivity to glucocorticoids. Med Hypotheses 2011;77(02):188-191

29 Dong T, Zhi L, Bhayana B, Wu MX. Cortisol-induced immune suppression by a blockade of lymphocyte egress in traumatic brain injury. J Neuroinflammation 2016;13(01):197. Doi: 10.1186/s12974-016-0663-y

30 Okazaki K, Kondo M, Kato M, et al. Serum cytokine and chemokine profiles in neonates with meconium aspiration syndrome. Pediatrics 2008;121(04):e748-e753 